\title{
Geociênciecias
}

\section{Metodologia para determinar parâmetros petrofísicos de corte em um campo de petróleo: o caso Socororo, bacia do Oriente, Venezuela}

\author{
Methodology to determine cut-off \\ petrophysical parameters: Socororo \\ case study, Eastern Venezuela Basin
}

Sérgio A. Cáceres Contreras

Engenheiro Geofísico,

Pesquisador da UNESPetro,

convênio UNESP-PETROBRAS,

Campus de Rio Claro, SP.

sacc@rc.unesp.br

\section{Joel Carneiro de Castro}

Professor Adjunto

Departamento de Geologia Aplicada, Universidade Estadual Paulista,

Campus de Rio Claro, SP.

jocastro@rc.unesp.br

\section{Resumo}

A potencialidade de produção de um reservatório petrolífero depende de alguns parâmetros de rocha, no caso dos parâmetros petrofísicos, sendo que os mesmos devem ser analisados antes de uma nova locação. Ao longo dos anos, algumas metodologias têm sido aplicadas com a finalidade de se determinarem os valores petrofísicos (mínimos e máximos) que garantam a produção comercial de um campo de petróleo. Nesse sentido, o presente trabalho expõe a descrição de um processo comumente usado na indústria de petróleo, o qual define os valores-limites desses parâmetros físicos da rocha e do fluido, os quais permitem avaliar se uma área é economicamente viável. O processo inclui o cálculo de parâmetros de corte, sendo que os mesmos são definidos pela resistividade mínima de produção do campo ou análogo, saturação máxima de água, saturação de água irredutível, volume máximo de argila e porosidade mínima. Uma vez determinados esses parâmetros, através do uso de gráficos cruzados, os mesmos são comparados com os valores medidos na região avaliada; caso algum deles se encontre fora dos limites mínimos ou máximos calculados, implicará alto risco econômico para a nova locação.

Palavras-chave: Parâmetro de corte, resistividade, porosidade, volume de argila, saturação de água.

\begin{abstract}
The production potential of a petroleum reservoir depends on rock parameters, such as petrophysical ones that must be analyzed before establishing a new location. For years some methods have been applied to define minimum and maximum petrophysical values and therefore to assure production from an oil field. The current research investigates a process commonly used in the oil industry that defines the boundary values of the rock and fluid parameters to assess the economic viability in an area. The process includes cut-off parameters, which are defined by the minimum resistivity for field production, maximum water saturation, irreducible water saturation, maximum clay content and minimum porosity. Once these parameters are defined through cross-plots, they are compared with measured values in the area; if some value is out of the calculated minimum or maximum limits, it will imply in considerable risk to the new location.
\end{abstract}

Keywords: Cut-off parameter, resistivity, porosity, shale volume, water saturation. 


\section{Introdução}

Para se avaliar uma oportunidade exploratória, é necessário determinar se a mesma poderia ter sucesso econômico. Neste sentido, existe uma metodologia baseada em análise petrofísica do reservatório, que permite determinar parâmetros de rocha mínimos e máximos capazes de predizer se a oportunidade tem chance de ser economicamente viável ou não.

Como exemplo para se explicar essa metodologia, serão utilizados dados de um campo de petróleo maduro, Socororo, localizado na região sudoeste da bacia Oriental da Venezuela/Sub-bacia Maturin (Figura 1). O campo é considerado como escola e tem sido operado pela empresa mista Petro-UCV desde 2000.

A exploração do campo Socororo foi iniciada no ano de 1939, sendo que, por problemas operacionais dos poços $\mathrm{e}$ da falta de compreensão da geologia do reservatório, a área foi abandonada. No ano 2000 , quando se reiniciou o processo de produção, o campo foi considerado como marginal, devido a sua baixa produção ( 200 barris por dia).

Até hoje o campo Socororo não produziu os volumes originalmente calculados, o que infere que o campo necessita de ter seu potencial reavaliado. A dificuldade apresentada pelo campo ocorre em função de os seus dados de poço serem antigos e de baixa qualidade e, ainda, de seus dados sísmicos serem limitados a algumas linhas sísmicas. $\mathrm{O}$ fato de o campo não ter atingido as condições de produção previamente calculadas indica que o mesmo ainda tem potencial. Os dados sísmicos e de poço têm mais de 30 anos de existência e são as únicas informações atuais representativas das condições de subsuperficie da área (Contreras, 2008).

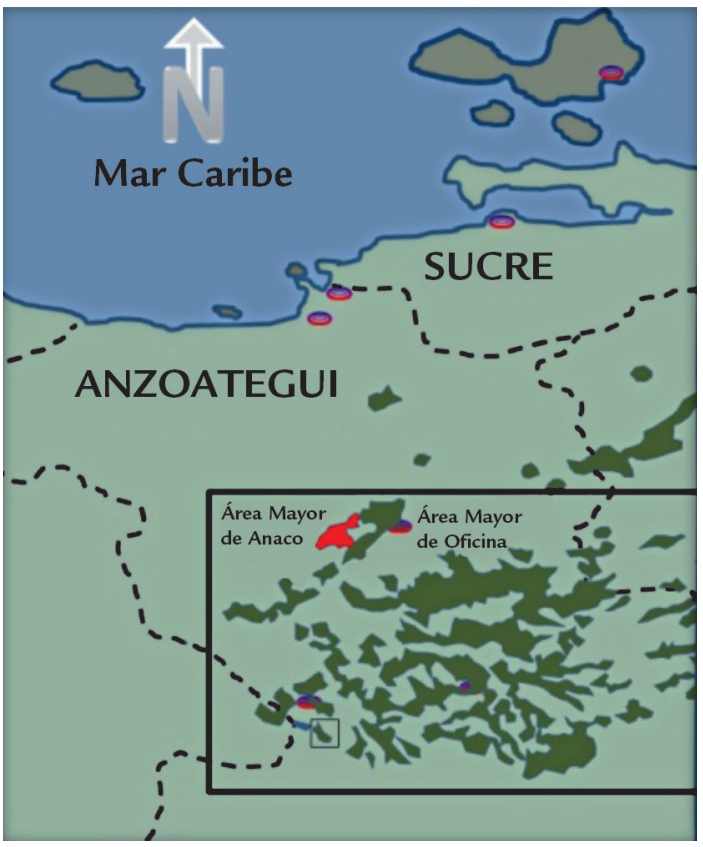

Para avaliar um perfil de poço (well-log), na ausência de amostras de rochas (testemunhos), é preciso analisar a sua correlação com perfis de outros poços. A correlação de perfis permite observar as variações litofaciológicas dos corpos sedimentares, o que facilita a interpretação geológica dos tipos de ambiente sedimentar nos níveis de possíveis reservatórios de hidrocarboneto.

A importância da definição de parâmetros de corte por reservatório (volume de argila máximo, porosidade mínima, saturação de água máxima, saturação de água irredutível, entre outros) é baseada na necessidade de indicar os valores mínimos e máximos dos mesmos, com os quais um intervaloreservatório pode ser considerado com potencial econômico suficiente para justificar a produção de hidrocarboneto do mesmo.

\section{Considerações petrofísicas}

A petrofísica consiste num sistema desenhado para a avaliação conjunta da rocha e dos fluidos, sendo que todas as considerações aqui realizadas utilizam as bases de cálculo propostas pela Schlumberger (1975) e por Bassiouni (1994).

O sistema de avaliação petrofísica é constituído, principalmente, por quatro elementos: litologia da rocha, volume de argila (Vsh), espaço vazio (porosidade) e fluido contido nos poros da rocha (óleo e/ou água).

Quando perfurado um poço, é de especial importância a avaliação do tipo de fluido presente nas camadas porosas e permeáveis da subsuperficie (Winsaucer, 1952). Dos fluidos, os registros medem saturações, enquanto o sistema rochafluido mede propriedades físicas, como resistividade, volume de argila (Vsh), porosidade $(\Phi)$ e permeabilidade (K). Esta última, por ser considerada uma propriedade dinâmica da rocha, só indica permeabilidade no instante de ser adquirido o registro; no caso de se verificar a permeabilidade é mantida ao longo do tem-
Figura 1

Localização do campo Socororo (quadro menor), a sudoeste da Área/ Província Oficina (quadro maior). Na cor vermelha, os campos de produção de gás e, na verde, os de produção de óleo. po, deve-se medir aquele parâmetro em múltiplas ocasiões.

O volume total de espaço poroso interconectado é denominado porosidade efetiva $(\Phi e)$, que em rochas arenosas é igual à porosidade total $(\Phi)$. No caso em que esses poros não estejam interconectados, não existirá permeabilidade.

O espaço poroso ocupado por volume de fluido é expresso em termos de saturação (\%); em outras palavras, um reservatório estará constituído por suas saturações de água $(\mathrm{Sw})$ e de hidrocarbo- 
neto (Sh). Deve-se esclarecer que a saturação de água é integrada pela saturação de água irredutível Swi (aquela que não fluirá quando a jazida entrar em produção) e pela saturação de água móvel Smo.

$\mathrm{Na}$ avaliação de perfis de poço, é considerada a interação da lama de perfuração com as paredes do poço, isto por causa da permeabilidade dos níveisreservatório ( zonas-reservatório); nesses níveis acontece uma interação de fluidos,

Partindo do Fator de Formação (F), Archie relacionou a resistividade da rocha preenchida com água (Ro) e a porosidade e mais dois parâmetros conhecidos como Coeficiente de Cimentação (m) e Coeficiente de Tortuosidade (a), os quais são calculados em laboratórios de rocha e mudam de uma bacia para outra $(\mathrm{F}=\mathrm{a} /$ $\left.\mathbb{\nabla}^{\mathrm{m}}\right)$. Os valores usados no campo Socororo são $\mathrm{a}=0.81$ e $\mathrm{m}=2$, os quais provêm

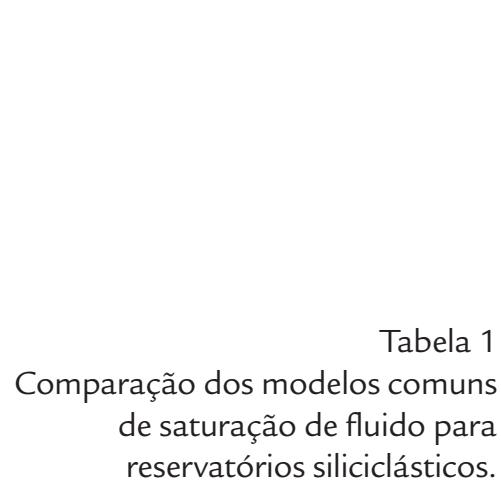

\section{Análise petrofísica e resultados}

A caracterização petrofísica é realizada nos níveis-reservatório e, por convenção, as siglas utilizadas, ao longo do artigo, respeitam seu nome em inglês, definido pelo American Petroleum Institute. Por exemplo, volume de argila tem devendo-se conhecer os parâmetros de rocha que podem ser medidos em cada uma das zonas. No processo de perfuração, as partículas sólidas da lama ficam nas paredes do poço, formando a zona de reboco ou mudcake, enquanto que a parte líquida da lama (filtrado) invade os níveis permeáveis por causa da pressão hidrostática da lama.

Um elemento importante de se mencionar é a resistividade da água de forma-

$$
\mathrm{F}=\mathrm{Ro} / \mathrm{Rw}
$$

das análises petrofisicas da região.

Outro parâmetro importante é o cálculo da Saturação, que classifica o conteúdo de fluido em função da argilosidade dos níveis-reservatório. Os modelos mais conhecidos e que se adaptam ao campo em estudo são os modelos de saturação de Archie (1941), Simandoux e Waxman-Smith. Cada um destes modelos é especificado na Tabela 1 , que inclui as ca- ção $(\mathrm{Rw})$, parâmetro que é utilizado para calibrar o conteúdo de fluido nos intervalos-reservatório, determinando e separando níveis que contêm água dos níveis que contêm óleo e/ou gás. O elemento Rw foi utilizado pela primeira vez por Archie (1941), ao definir o Fator de Formação (F), que é a relação que existe entre a resistividade da rocha preenchida com água (Ro) e a resistividade da água de formação $(\mathrm{Rw})$, como apresentado na equação 1 .

racterísticas básicas que deve ter a rochareservatório para se decidir se é aplicado um ou outro modelo de saturação.

Deve-se lembrar que os valores calculados de saturação de fluido e permeabilidade são os que existiam no campo, na data em que foi adquirida a informação; caso o campo ou jazida tenha entrado em produção, esses valores estarão alterados.

\begin{tabular}{|c|c|c|c|c|}
\hline Modelo de & $\%$ de & \multicolumn{3}{|c|}{ Características relevantes } \\
\hline Archie (1941) & $\begin{array}{c}\text { Menor } \\
\text { que } 20 \% .\end{array}$ & \multirow{2}{*}{$\begin{array}{c}\text { Não considera } \\
\text { tipos } \\
\text { de argila }\end{array}$} & $\begin{array}{c}\text { Independente da } \\
\text { porosidade. }\end{array}$ & $\begin{array}{l}\text { Requer os coeficientes } \\
\text { a e } \mathbf{~ m .}\end{array}$ \\
\hline Simandoux & \multirow{2}{*}{$\begin{array}{c}\text { Maior } \\
\text { que } 20 \% .\end{array}$} & & $\begin{array}{c}\text { Não trabalha com } \\
\text { porosidades menores } \\
\text { que } 20 \% .\end{array}$ & $\begin{array}{l}\text { Não requer análise de } \\
\text { testemunho, mas, sim, } \\
\text { dos coeficientes a e m. }\end{array}$ \\
\hline $\begin{array}{l}\text { Waxman- } \\
\text { Smith }\end{array}$ & & $\begin{array}{c}\text { Considera } \\
\text { tipos } \\
\text { de argila }\end{array}$ & $\begin{array}{c}\text { Trabalha com } \\
\text { porosidades menores } \\
\text { que } 20 \% .\end{array}$ & $\begin{array}{l}\text { Requer análise de } \\
\text { testemunho para determinar } \\
\text { o coeficiente de intercâmbio } \\
\text { eletrolítico. }\end{array}$ \\
\hline
\end{tabular}

a sigla Vsh (shale volume).

Para iniciar a avaliação petrofísica devem ser predeterminados alguns parâmetros, entre eles o modelo matemático de saturação de fluido (que será utilizado nos cálculos de conteúdo de fluido), a calibração dos registros elétricos, para o cálculo do tipo de fluido, e a utilização dos coeficientes de tortuosidade e cimentação $(\mathrm{a}, \mathrm{m})$ próprios da bacia. Neste sentido, o processo de análise petrofísica será resumido a seguir.

\section{Seleção do modelo de saturação de fluido}

O modelo de saturação utilizado depende do conteúdo de argila nos níveis-reservatório de interesse. Para a realização dessa análise, é calculado o volume de argila (Vsh) nos perfis de Potencial Espontâneo (SP) e/ou Raio Gama (GR), este nem sempre disponível no campo Socororo (Figura 2). Para o cálculo de volume de argila, foi usado o índice de argilosidade (Ish), que é uma aproximação ao valor real do volume. Os valores resultantes desse cálculo mostram um porcentual de argila acima do valor real, sendo que existem duas fórmulas propostas pela Dresser Atlas (in Rider,1986) para o ajuste do valor:
Vsh $=0.33\left(2^{2 \text { Ish }}-1\right)$, em rochas pré-

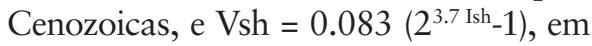
rochas Cenozoicas.

O volume médio de argila é de $21 \%$, para os três intervalos-reservatório do poço 46. A equação utilizada pelo programa Prizm foi baseada no registro de Raio Gama:

$$
\text { Volume de Argila (Vsh) }=[(\text { GR-GRcln) } /(\text { GRsh-GRcln })]
$$

Sendo: GR = medida do perfil Raio Gama, no intervalo de interesse, e GRcln e GRsh
= média do valor de Raio Gama, nos arenitos (cln, clean) e nos folhelhos (sh).
A partir da análise anterior, repetida para um total de 15 poços e média de 
cinco intervalos-reservatório para cada poço, determinou-se que a equação de saturação a ser utilizada, nesse projeto, é a de Simandoux, pois o conteúdo de argila, nos reservatórios, está acima de $20 \%$ e não se dispõe do valor de coeficiente de intercâmbio eletrolítico, que permitiria aplicar a equação de Waxman-Smith.

$\mathrm{O}$ processo anterior é realizado com o objetivo de definir o conjunto de equações mais apropriado para calcular o modelo de saturação de fluido nos

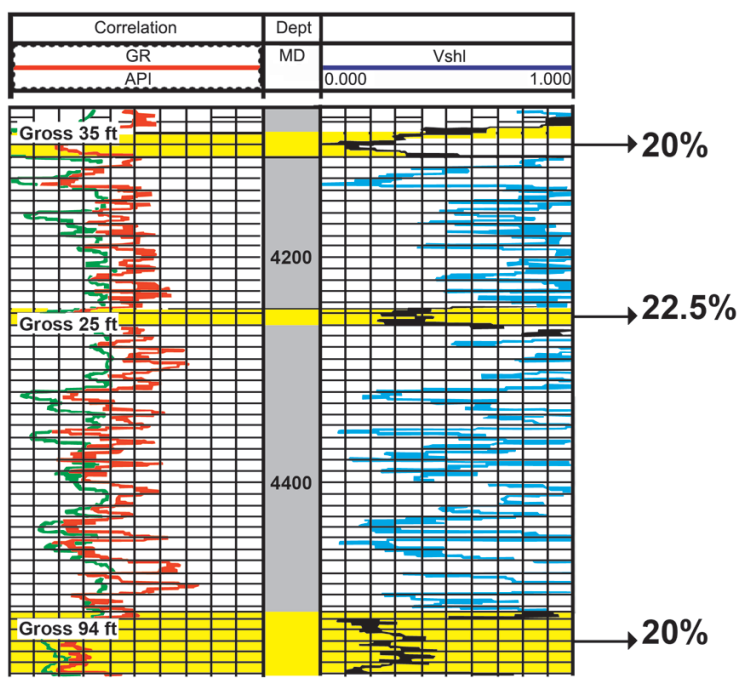

\section{Calibração dos registros elétricos para cálculo de fluido}

Para se determinar o conteúdo de fluido (gás, óleo ou água) presente nos níveis-reservatório, deve-se calibrar o registro elétrico de Potencial Espontâneo (SP), através da seleção de um nível arenoso com mais de 20 pés de espessura ( 6 metros), e preenchido com água, de preferência água salgada. A determinação desse nível é realizada com base nos perfis de resistividade, como o registro de ILD (Induction dual laterolog).

Nessa fase, tem de se definir o valor da resistividade da água de formação $(\mathrm{Rw})$ do nível selecionado, o qual foi calculado, nesse projeto, a partir do registro de SP do poço 46 (Figura 2). No mesmo poço, o melhor nível arenoso se encontra entre 3713 pés e 3735 pés (Figura 3A), com uma resistividade de 2,44 ohm_m@ $57,95^{\circ} \mathrm{C}$.

Para o cálculo de Rw, são necessários os seguintes parâmetros extraídos do cabeçalho do registro de poço (dados do poço 46):

- Resistividade do filtrado de lama de perfuração (Rmf) na temperatura de superfície $\left(\mathrm{T}_{\mathrm{s}} 27,78^{\circ} \mathrm{C}\right): \mathrm{Rmf} @ \mathrm{~T}_{\mathrm{s}}$ $=1,2$ Ohm . m @ 27,78 ${ }^{\circ} \mathrm{C}$.

- Profundidade final BHD (Bottom Hole Depth), 4806 pés.

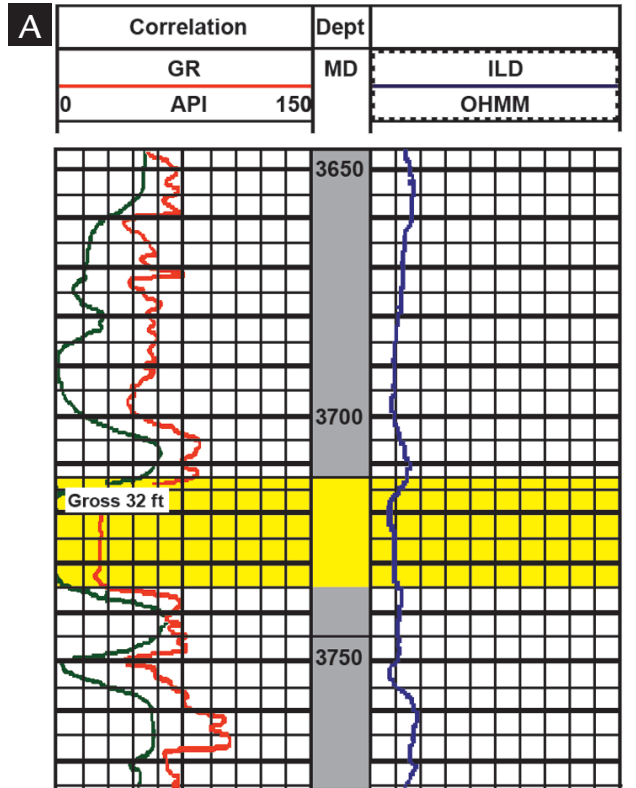

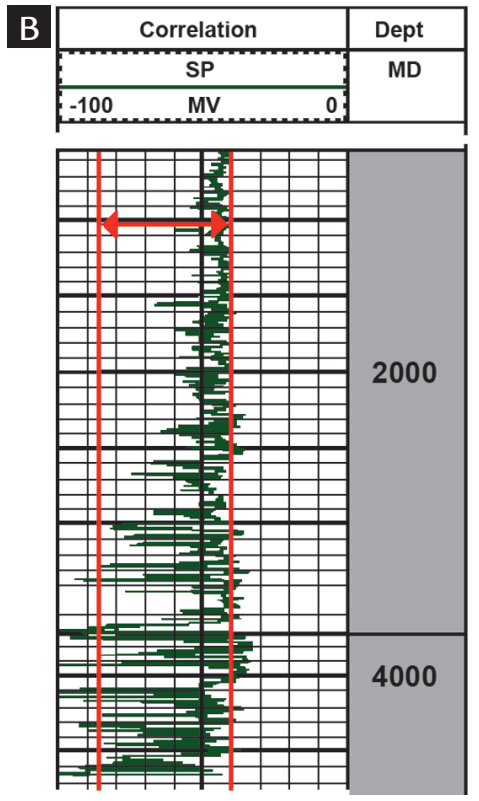

níveis-reservatório. Esses parâmetros são fundamentais na seleção do conjunto de equações a partir do qual serão calculadas as propriedades físicas do reservatório, tais como porosidade, areia total, areia petrolífera e teor de fluido.
Figura 2

Cálculo de volume de argila (Vsh) para três intervalos arenosos do poço 46. Curva Potencial Espontâneo SP (verde) à esquerda do Raio Gama. Observam-se três medidas da média de Vsh, para cada intervalo-reservatório (lado direito da figura; profundidade em pés).
- Temperatura do fundo do poço BHT (Bottom Hole Temperature), $66,67^{\circ} \mathrm{C}$.

- Gradiente de temperatura, $\mathbf{g}_{\mathrm{G}}$ 0,00809 ${ }^{\circ} \mathrm{C} /$ pé.

Os valores anteriores são complementados por outros, como o SSP, calculado no poço 46. O valor de SSP (diferença em milivolts entre a linha-base dos arenitos e dos folhelhos) foi medido no perfil SP, onde a linha-base de folhelhos está à direita e a de arenitos à esquerda, dando um resultado de $45 \mathrm{mV}$ (Figura 3B). Deve-se lembrar que a deflexão da linha de arenito para a esquerda é pelo fato de a lama de perfuração ser menos
Figura 3

A) Melhor nível arenoso selecionado para o cálculo de Rw (a curva verde corresponde ao perfil SP), com espessura de 22'.

B) Cálculo de SSP para o poço 46. 
salina que a água da formação. Quando a deflexão dessa linha é para a direita, interpreta-se que a lama de perfuração é

Sendo:

$\mathrm{T}_{f}$ (temperatura da formação).

SSP = diferença em milivolts entre a deflexão das linhas-base de folhelho e de arenito.

$\mathrm{K}=$ constante que depende da temperatura da formação.

O resultado do cálculo dos parâmetros, para a obtenção de Rw, para o intervalo entre 3713'e 3735', indicou $\mathrm{T}_{\mathrm{f}}=$ $57,95^{\circ} \mathrm{C}$; $\mathrm{K}=68,71$; e SSP=45 mV.

A resistividade do filtrado de lama (Rmf), na temperatura da formação, foi calculada no gráfico da Schlumberger (1975), apresentado na Figura 4. Nes- mais salina que o fluido da formação.

Os dados anteriores são complementados por outros, calculados e es-

$$
\begin{gathered}
R w=\left(R m f @ T_{f}\right) / 10^{S S P / K} \\
T_{f}=T_{s}+\left(g_{G} \times \text { Prof }_{f}\right)
\end{gathered}
$$

se gráfico se ingressa com os valores de Rmf @ Ts (1,2 ohm.m @ 27,78 C),com o objetivo de se calcular o conteúdo de $\mathrm{NaCl}$ em partes por milhão, no caso 4300 ppm, o qual não muda conforme a temperatura (a resistividade depende da temperatura). A seguir, sobre o gráfico, é projetada uma linha reta. $\mathrm{Na}$ referida linha, um ponto representa o valor da temperatura de formação e o outro ponto o conteúdo de $\mathrm{NaCl}$ (em ppm), donde se obtém o valor procurado, $\mathrm{Rmf} @ \mathrm{~T}_{\mathrm{f}}(0,75$ ohm.m@57,95 $\mathrm{C}$ ).

Com esses valores, em conjunto com a equação (3), se obtém a primeira pecificados na Tabela 2. Nesse sentido, Rw será calculado com a seguinte equação:

aproximação do Rw, resultando em 0,17 ohm.m@57,95 ${ }^{\circ} \mathrm{C}$; logo após, esse valor tem de ser ajustado com o Pickett Plot, gráfico com dois eixos logarítmicos, porosidade $(\mathrm{Y})$ versus resistividade $(\mathrm{X})$ e construído dentro do programa Prizm (Figura 5).

Para se realizar o ajuste com o Pickett Plot (Pickett, 1966), são carregadas as condições-limite (boundary conditions; ver Tabela 2).

São carregadas as curvas de indução ILD (Induction Dual Laterolog) versus PHIN (Porosidade Neutrônica). O crossplot inclui saturação de água ajus-
Figura 4 Cálculo de Rmf à temperatura da formação.
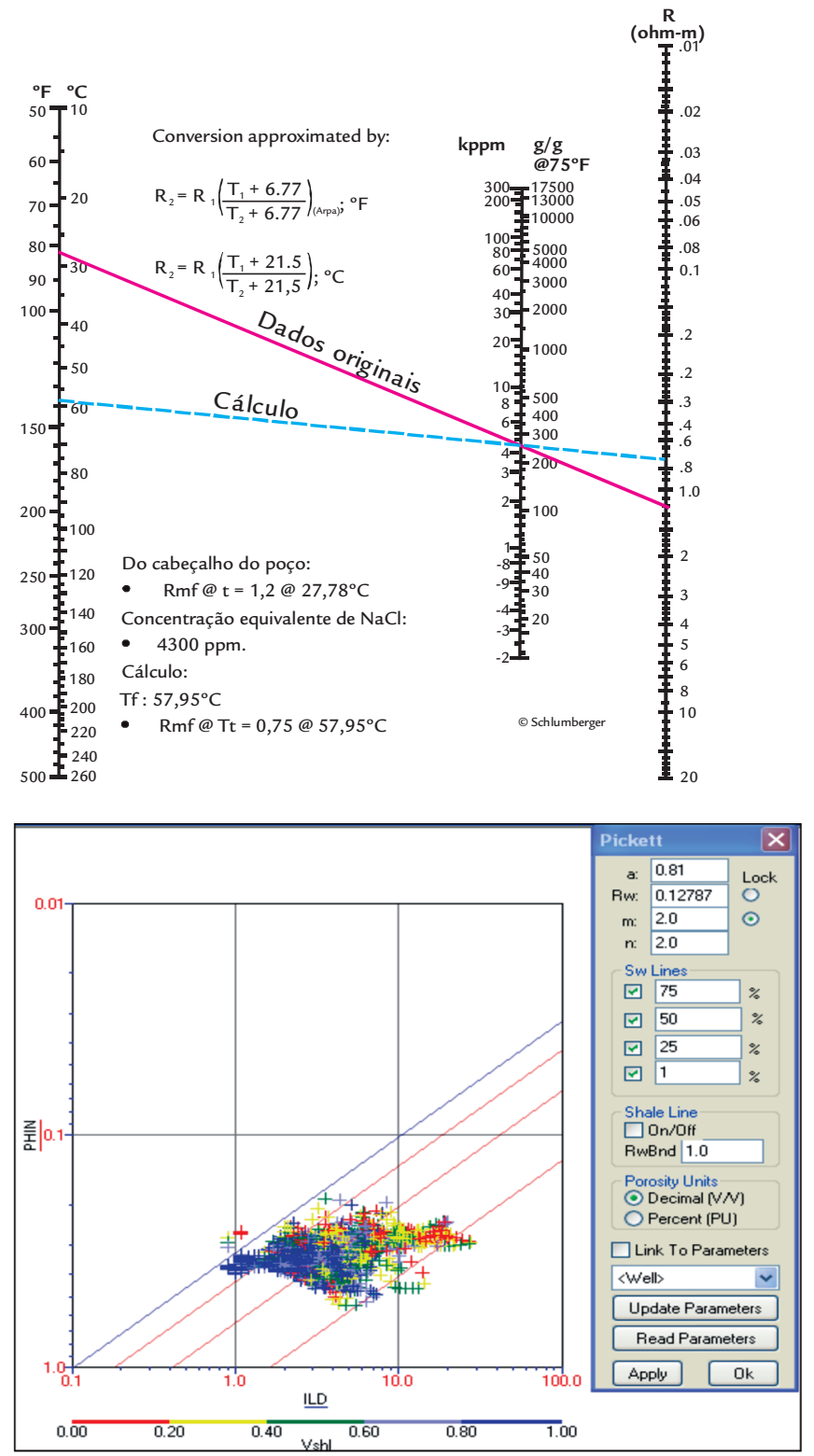

Pickett Plot com o ajuste do Rw. As cruzes em cor vermelha apresentam o menor conteúdo do Vsh e, em azul, o maior.

As linhas inclinadas representam saturação de água (linha azul aumentando no sentido das vermelhas). 
Set Parameters - Archie Interpretation

$\mathrm{X}$

\begin{tabular}{|c|c|c|c|c|c|}
\hline Parameters & Zone Definition & \multicolumn{2}{|c|}{ Zoned WellParameters } & & Raio gama limpo (ex. arenito) \\
\hline Name & Source & Value & Unit & Description & \multirow{2}{*}{ Raio gama folhelho } \\
\hline GRclr & UDE Set & 22.000 & API & Gamma ray clean & \\
\hline GRshl & UDE Set & 80.000 & API & Gamma ray thale & \multirow{2}{*}{ Neutrão - Índice de hidrogênio do folhelho } \\
\hline HIshl & UDE Set & 0.400 & $\mathrm{~V} / \mathrm{V}$ & Neutron hydrogen index shale & \\
\hline RhoF & UDE Set & 1.000 & $\mathrm{GM} / \mathrm{CC}$ & Bulk density fluid & \multirow[b]{2}{*}{ Densidade de massa do fluido } \\
\hline $\begin{array}{l}\text { RhoM } \\
\text { RhoShl }\end{array}$ & UDE Set & 2.650 & $\mathrm{GM} / \mathrm{CC}$ & \begin{tabular}{|l|} 
Bulk density matrix \\
Bulk density shale
\end{tabular} & \\
\hline Rshl & UDE Set & $\begin{array}{l}2.400 \\
5.000\end{array}$ & \begin{tabular}{|l}
$\mathrm{GM} / \mathrm{CC}$ \\
$\mathrm{OHMM}$
\end{tabular} & \begin{tabular}{|l|} 
Bulk density shale \\
Resistivity shale
\end{tabular} & \multirow{2}{*}{ Densidade de massa da matriz } \\
\hline $\mathrm{Rw}$ & UDE Set & 0.170 & OHMM & Formation water resistivity & \\
\hline $\mathrm{a}$ & UDE Set & 0.810 & & Archie "a" & \multirow{2}{*}{ Densidade de massa do folhelho } \\
\hline $\mathrm{m}$ & UDE Set & 2.000 & & Archie "m" & \\
\hline $\mathrm{n}$ & UDE Set & 2.000 & & Archie "n" & Resistividade do folhelho \\
\hline & & & & & Resistividade da água da formação \\
\hline & & & Ok & Cancel & Help \\
\hline
\end{tabular}

tada ao valor de Rw=0,127 ohm.m @ $57,95^{\circ} \mathrm{C}$.

Observa-se que, na janela do programa, aparece interpretação do tipo Archie e não Simandoux, como foi estabelecido. O programa Prizm (Landmark Co.) é um dos poucos programas do mercado que inclui o modelo de saturação de Simandoux dentro do modelo Archie; isto acontece porque, na realidade, o modelo de saturação Simandoux é uma equação modificada do modelo de Archie. A diferença do Prizm, em comparação a outros programas, é que o modelo de saturação de Simandoux aparece como foi definido originalmente na área petrofísica. Neste sentido, Simandoux acrescentou a presença de volume de argila dentro da equação de Archie. No caso em que os volumes de argila são menores que $15 \%$ e próximos de zero, a equação de Simandoux é igual à equação de Archie; em outros programas de caracterização petrofísica, o modelo de Simandoux é considerado um modelo de saturação independente.

O cálculo de conteúdo de fluido provém da interpretação dos registros elétricos e, em geral, o fluido obedece a valores de resistividade-padrão: 0.000000001 a 2 ohm.m, água salgada; 1 a 10 ohm.m, água doce; 50 a 150 ohm.m, petróleo; e

\section{0 a >1500 ohm.m, gás.}

Compreende-se que a análise petrofisica termina, uma vez conhecidas as características físicas do reservatório calculadas para cada poço e integradas em mapas de isopropriedades (mapas de porosidade, volume de areia, areia oleígena total, entre outros).

Finalizada a análise petrofísica, é necessário o cálculo dos parâmetros de corte, que representam os valores máximos e mínimos permitidos para o campo. Nesta ordem de idéias, partindo-se do valor de resistividade mínima, para a produção de óleo, são calculadas a saturação de água irredutível (Swi) do reservatório e a saturação máxima de água (Sw) permitida para o mesmo. Incluindose o valor de saturação máxima de água num crossplot de Sw x Volume de argila (Vsh), obtém-se o valor máximo de argila permitido para a oportunidade. Finalmente, num crossplot Vsh x Porosidade, é calculada a porosidade mínima que o reservatório deve apresentar, para uma produção econômica.

Os resultados desses crossplots são apresentados a seguir. No primeiro gráfico, ILD (resistividade) x saturação de água (Figura 6), se ingressa com o valor mínimo da resistividade de produção de hidrocarboneto no campo ( $\mathrm{Rt}=8$

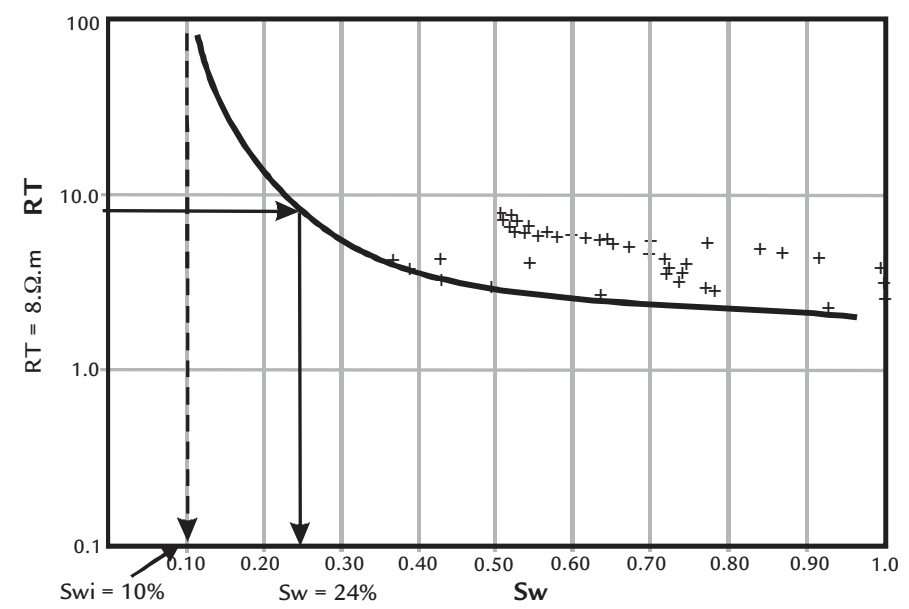

Tabela 2

Condições de contorno

para o Pickett Plot.

ohm.m) e se projeta esse valor sobre a linha-tendência (sub-horizontal) da nuvem de pontos gerados; dessa intersecção se projeta uma linha vertical até o eixo " $\mathrm{X}$ ", onde se mede a saturação máxima de água $(S w=24 \%$ ) para a qual esse reservatório produz. Sobre o mesmo gráfico, é calculada a saturação de água irredutível (Swi $=10 \%$ ), com uma linha paralela ao eixo "Y" (onde os dados tornam-se assintóticos ao referido eixo).

No segundo gráfico (Figura 7), são cruzados volume de argila x saturação de água. Nele se ingressa com valor da saturação de água ( $24 \%$, ver gráfico anterior) e, no ponto de intersecção da vertical de $36 \%$ com a diagonal média da "nuvem" de pontos, é determinado o volume máximo de argila que pode conter o reservatório (no caso, 24\%).

Já no último gráfico (Figura 8), é calculada a porosidade mínima de produção $(20 \%)$ do nível-reservatório, ingressando-se no gráfico com o valor de volume de argila (0.17) encontrado no gráfico prévio.

Assim ficam definidos os parâmetros de corte que garantem a produção comercial no campo: Vsh não pode ser superior a $17 \%$, a porosidade $(\Phi)$ deve ser acima de $20 \%$, Sw deve ser menor que $24 \%$ e Swi deve ser igual a $10 \%$.

Figura 6

Gráfico de resistividade $x$ saturação de água. 

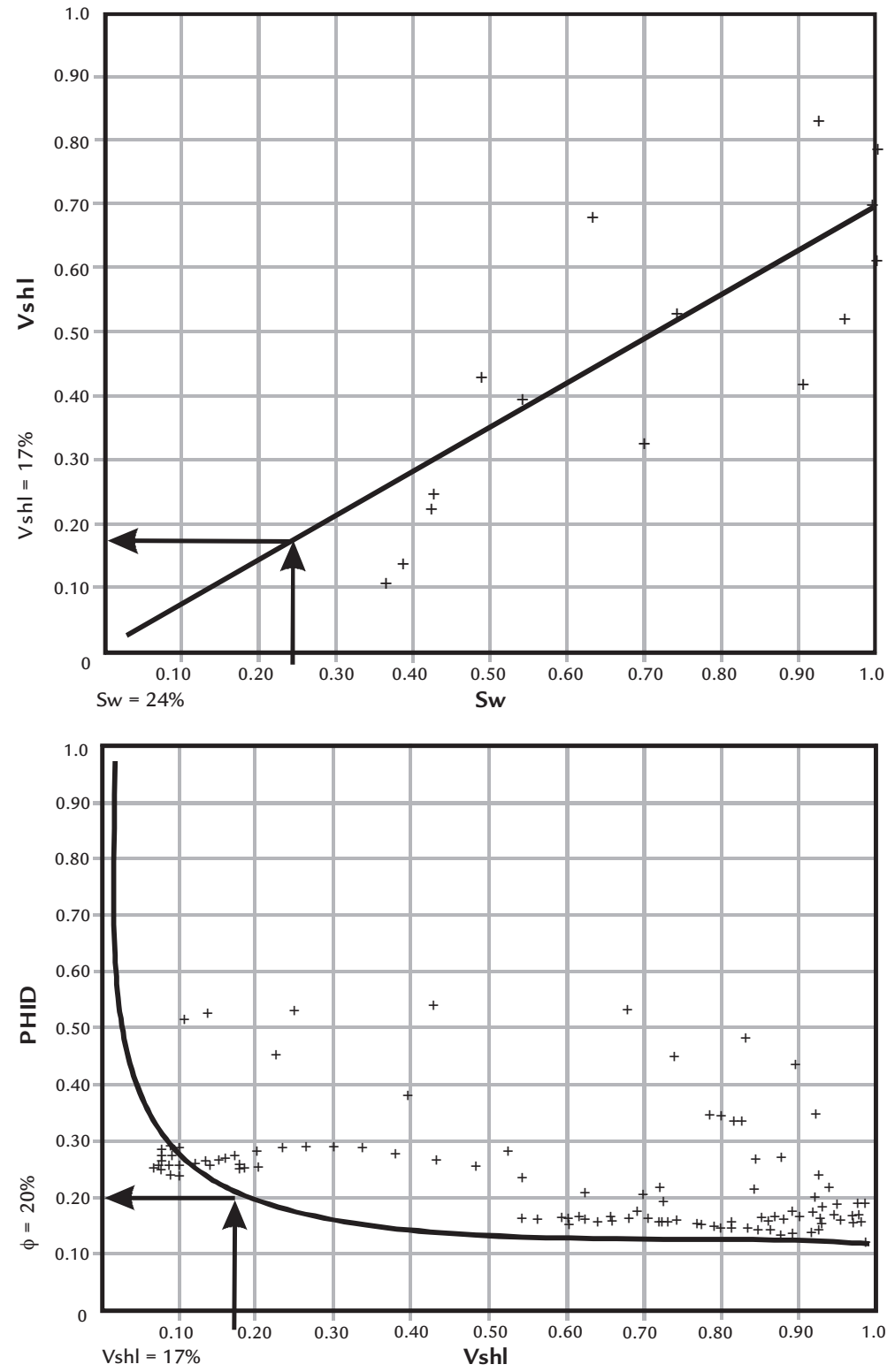

Figura 7

Gráfico de saturação de água $x$ volume de argila.

Figura 8

Gráfico volume de argila $x$ porosidade do neutrão.

\section{Conclusões}

Num potencial campo de petróleo, quando considerado o risco econômico, pode-se predizer a produção comercial do mesmo com certo grau de certeza. Neste sentido, os parâmetros petrofísicos calculados a partir das informações que provêm de geofísica de poço permitem mensurar as caraterísticas do reservatório. $\mathrm{O}$ fato anterior poderia contribuir na definição do nível de investimento necessário para se iniciar a produção comercial do poço e/ou do campo. Por outro lado, a necessidade de se aumentar a produção

\section{Agradecimentos}

Esse trabalho foi desenvolvido junto ao programa de pós-graduação em Geologia Regional, campus de Rio Claro da Universidade Estadual Paulista Júlio de Mesquita de um campo já descoberto faz necessária a perfuração de novos poços, os quais são avaliados e comparados a partir dos poços pioneiros. Essa avaliação torna-se mais eficiente quando valores de corte de produção são incluídos na análise, sendo que esses valores orientaram o reconhecimento de novas locações para continuar e/ou iniciar a produção de um campo.

Ao longo do exemplo desenvolvido nesse trabalho, o qual considera um campo maduro com problemas de fronteira exploratória e baixa recuperação do volume de hidrocarboneto originalmente calculado, conclui-se que os resultados confirmam a capacidade produtora do campo Socororo. Seus problemas de produção, principalmente associados à engenharia de perfuração, compensam ser solucionados, pois os parâmetros de corte garantem a produção comercial do mesmo. Tendo sido calculado que: o Vsh não pode ser superior a $17 \%$, a porosidade $(\Phi)$ deve ser acima de $20 \%$, a $\mathrm{Sw}$ deve ser menor que $24 \%$, e a Swi deve ser igual a $10 \%$.
Filho. O primeiro autor agradece ao programa CAPES pela bolsa de doutorado concedida. Agradecemos à Landmark Graphics Corporation pelo apoio mediante o fornecimen- to do programa Prizm (Landmark University Grant Program) e à Petro-UCV e a Universidade Central de Venezuela, que permitiram a utilização dos dados. 


\section{Referências bibliográficas}

ARCHIE G. Electrical resistivity $\log$ as an aid in determinig some reservoir characteristics. Petroleum Technology, 1941.

BASSIOUNI, Z. Theory, measurement, and interpretation of Well Logs. Soc. Petroleum Engineers, Richardson, TX, p 371. 1994.

CONTRERAS S. Modelagem 3D e identificação de ambientes sedimentares em registros de poço, nos níveis das formações Oficina e Merecure do campo Socororo. (Estado Anzoátegui - Venezuela). Universidade Estadual Paulista Júlio de Mesquita Filho, 2008. 154p. (Tese de Doutorado).

RIDER, M. H. The geological interpretation of Well Logs. Blackie and Son Limited, 1986. 175p.

SCHLUMBERGER Interpretación de Perfiles. Fundamentos Schlumberger Limited, 1975. v. 1. 155p.

WINSAUCER $\mathrm{H}$, et al. Resistivity of Brine-Saturated Sands in relation to pore geometry. AAPG Bulletin, v. 36, n. 2, p. 253-277, 1952.

Artigo recebido em 19 de setembro de 2011. Aprovado em 11 de junho de 2012. 